\section{The National Respiratory Research Strategy - a key Canadian Thoracic Society priority}

$\mathrm{T}$ he Canadian Thoracic Society (CTS) Executive Committee never has a meeting without talking about research, and that's because research matters... a great deal. Not only is research one of the CTS' pillars (research, guidelines, education and knowledge translation), but it is also a key component of our efforts with our partners to make a difference in respiratory health. It's no surprise then that the National Respiratory Research Strategy (NRRS) is at the top of the CTS agenda.

A short while ago, Denis O'Donnell, former CTS President, reported that directors of academic respiratory divisions in Canada "overwhelmingly indicated their concern about the current erosion of clinical research in Canada and the progressive decline in funding, infrastructure and training opportunities" (1). He was clear at that time that "urgent steps need to be taken to address these very real concerns and to preserve our well-deserved reputation for excellence in clinical research in respiratory diseases" (1).

Well, urgent steps have been taken. CTS has led the way, with the Canadian Lung Association (CLA), in developing the NRRS. We are well on our way to implementing the main components of the strategy, the Respiratory National Scientist Core Education and Training (RENASCENT) Program and the Canadian Respiratory Research Network (CRRN).

The RENASCENT Program will promote interdisciplinary research and professional skills development, offer mentoring, foster the development of networking and team building skills, and support trainees and new investigators at transition points in career development. The program will direct its efforts to supporting trainees across all four Canadian Institutes of Health Research (CIHR) pillars of clinician- and nonclinician-researchers at the graduate, postdoctoral and new investigator level.

The CRRN will bring together researchers and research platforms to facilitate innovative and collaborative research in respiratory health. The goals of the CRRN are to integrate research efforts, create added value and answer research questions that may only be addressed by a broad network. The CRRN team agreed to build the network on current strengths and reached consensus on the theme of 'origins and progression of airway diseases'. The focus will be on the two most common lung diseases in Canada - asthma and chronic obstructive pulmonay disease, areas in which we have expertise and credibility.

The CRRN will also translate and apply research results to reduce the economic and health burden of respiratory diseases. It will strengthen alliances and networking opportunities with national, provincial and international partners, and it will include early career support for young investigators while enhancing collaborations with Canadian institutions. The CRRN will eventually provide platforms and infrastructure for future networked projects that will focus on other lung diseases.

Together, the RENASCENT Program and the CRRN will begin to address the gaps so aptly highlighted by Dr O'Donnell. We recently submitted a letter of intent to the CIHR in the hope of securing funding for the CRRN and the CLA Board of Directors approved the use of $\$ 1.085$ million of research reserves over five years (as $50 \%$ of the required matching partnered funding) for the CRRN. They also

\section{La Stratégie nationale de recherche en santé respiratoire - une priorité clé de la Société canadienne de thoracologie}

I e Comité exécutif de la Société canadienne de thoracologie (SCT) Lne tient jamais de réunion sans discuter de la recherche, car la recherche revêt une énorme importance. C'est non seulement l'un des piliers de la SCT (recherche, lignes directrices, formation et application du savoir), mais également un aspect clé des efforts avec les partenaires pour changer les choses en santé respiratoire. On ne s'étonnera donc pas que la Stratégie nationale de recherche en santé respiratoire (SNRSR) figure au sommet du programme de la SCT.

Dernièrement, Denis O'Donnell, ancien président de la SCT, a signalé que les directeurs des départements universitaires de pneumologie du Canada étaient « très inquiets de l'érosion actuelle de la recherche clinique au Canada et du déclin progressif de son financement, de ses infrastructures et des possibilités de formation dans ce domaine » (1). Il a clairement affirmé que " des mesures urgentes s'imposent pour redresser cette situation très préoccupante et pour préserver notre réputation bien méritée d'excellence en recherche clinique sur les maladies respiratoires » $(1)$.

Des mesures urgentes ont bel et bien été prises. Conjointement avec l'Association pulmonaire du Canada (APC), la SCT a dirigé la création de la Stratégie nationale de recherche en santé respiratoire (SNRSR). Nous sommes sur le point d'en lancer les premiers éléments : le programme RENASCENT (Respiratory National Scientist Core Education and Training, ou Enseignement et formation de base en santé respiratoire aux scientifiques à l'échelle nationale) et le Réseau canadien de recherche en santé respiratoire (RCRSR).

Le Programme RENASCENT fera la promotion de la recherche interdisciplinaire et de l'acquisition de compétences professionnelles, fournira du mentorat, favorisera l'acquisition de compétences de réseautage et de renforcement de l'esprit d'équipe et appuiera des stagiaires et de nouveaux chercheurs à des moments décisifs de leur carrière. Le programme appuiera des stagiaires cliniciens et non cliniciens aux études doctorales ou postdoctorales ainsi que de nouveaux chercheurs dans les quatre piliers des Instituts de recherche en santé du Canada (IRSC).

Le RCRSR rassemblera des chercheurs et des plateformes de recherche pour faciliter la recherche novatrice et concertée en santé respiratoire. Il cherchera ainsi à intégrer les efforts de recherche, à créer une valeur ajoutée et à répondre aux questions de recherche que seul un vaste réseau est en mesure d'aborder. L'équipe du RCRSR a convenu de bâtir le réseau d'après les forces actuelles et de miser sur les origines et la progression des maladies des voies respiratoires. Le RCRSR mettra l'accent sur les deux principales maladies respiratoires au Canada, l'asthme et la maladie pulmonaire obstructive chronique, à l'égard desquelles le Canada possède des compétences et une crédibilité démontrées.

De plus, le RCRSR transférera les résultats de la recherche et les mettra en pratique, de manière à réduire le fardeau économique et sanitaire des maladies respiratoires. Il renforcera les alliances et les occasions de réseautage avec des partenaires nationaux, provinciaux et internationaux et offrira un soutien aux jeunes chercheurs en début de carrière, tout en accroissant les collaborations avec des établissements canadiens. Il fournira ensuite des plateformes et des infrastructures aux projets réseautés qui porteront sur d'autres maladies respiratoires. 
approved use of research reserves this fiscal year to enable the National Research Steering Committee to design the implementation of the NRRS, which includes measuring our fundraising readiness and preparing plans for a multiphase campaign. This is a great start that will allow us to leverage additional support for the NRRS. However, it is vital that, as part of the restructuring process that it is currently undergoing, the CLA affirm once again, that research will remain a core priority for the association.

I am most grateful to our key partners, the CLA, the Canadian Respiratory Health Professionals and the CIHR, for the support they have shown for the NRRS. Thanks as well to past presidents, including Denis O'Donnell and George Fox, to CTS Research Committee Chair, Andrew Halayko and to CLA Director of Research \& Knowledge Translation Anne Van Dam and CTS Director Janet Sutherland, who have all contributed so much of their time and expertise to the development of the NRRS.

With their help, and that of all of our partners, we will succeed in keeping the NRRS at the top of our agenda - where it belongs. Why? Because the NRRS matters - a great deal - to CTS members and to respiratory health in Canada.

\section{REFERENCE}

1. O'Donnell DE. Prioritizing research at the Canadian Thoracic Society and beyond. Can Respir J 2010;17:9-10.

Respectfully submitted, Robin G McFadden MD FRCPC President, Canadian Thoracic Society
Ensemble, le programme RENASCENT et le RCRSR commenceront à corriger les lacunes qu'a si bien relevées le docteur O'Donnell. Nous avons récemment écrit une lettre d'intention aux IRSC dans l'espoir d'obtenir du financement pour le RCRSR, et le conseil d'administration de l'APC a approuvé l'utilisation de 1,085 million de dollars tirés de la réserve de recherche pour le RCRSR sur cinq ans, soit la moitié du financement de contrepartie requis. Il a aussi approuvé l'utilisation de fonds de la réserve de recherche pendant le présent exercice, afin de permettre au Comité directeur national de la recherche de planifier la mise en œuvre de la SNRSR, ce qui inclut la mesure de la préparation en matière de collecte de fonds et l'élaboration d'un plan de campagne réalisé par tranches. Il s'agit d'un excellent départ pour susciter des appuis supplémentaires à la SNRSR. Toutefois, dans le cadre du processus de restructuration actuel, il est crucial que l'APC réitère la place prépondérante qu'elle accorde à la recherche.

Je remercie sincèrement nos principaux partenaires (l'APC, les Professionnels canadiens en santé respiratoire [PCSR] et les IRSC), pour leur appui à la SNRSR. Je remercie également les anciens présidents de la SCT, notamment Denis O'Donnell et George Fox, le président du Comité de la recherche de la SCT, Andrew Halayko, la directrice de la recherche et du transfert des connaissances de l'APC, Anne Van Dam, et la directrice de la SCT, Janet Sutherland, qui ont consacré leur temps et leurs compétences à la mise sur pied de la SNRSR.

Grâce à leur aide et à celle de tous nos partenaires, nous réussirons à maintenir la SNRSR là où elle doit être : au sommet de notre programme. Pourquoi? Parce que la SNRSR revêt une énorme importance pour les membres de la SCT et pour la santé respiratoire au Canada.

\section{RÉFÉRENCE}

1. O'Donnell DE. Prioriser la recherche à la Société canadienne de thoracologie et au-delà. Can Respir J 2010;17:9-10.

Salutations distinguées, Robin G McFadden MD, FRCPC President, Société canadienne de thoracologie 


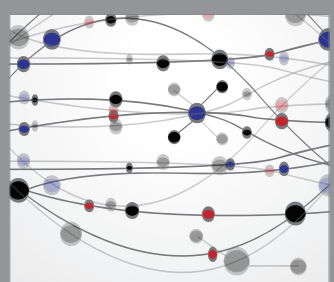

The Scientific World Journal
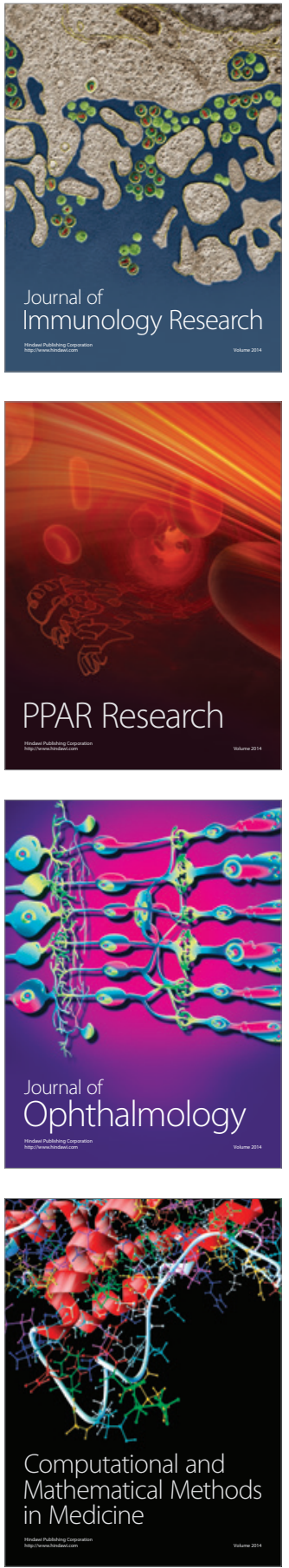

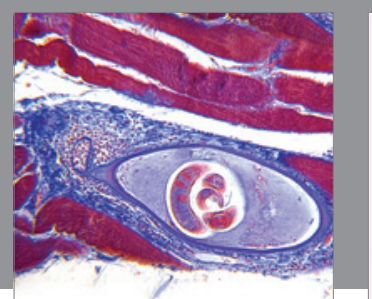

Gastroenterology Research and Practice

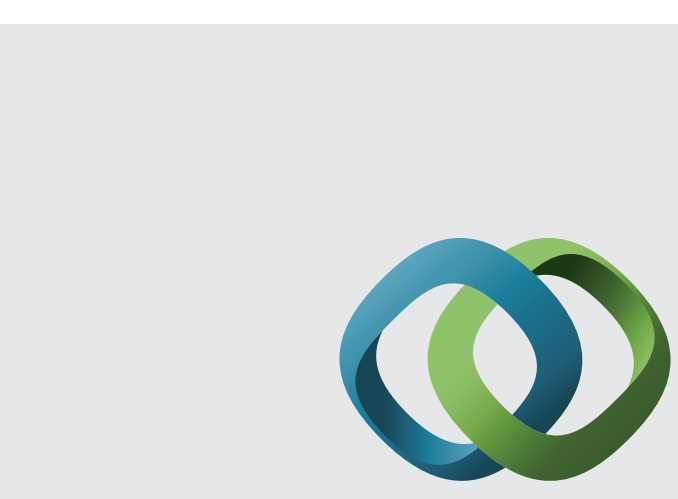

\section{Hindawi}

Submit your manuscripts at

http://www.hindawi.com
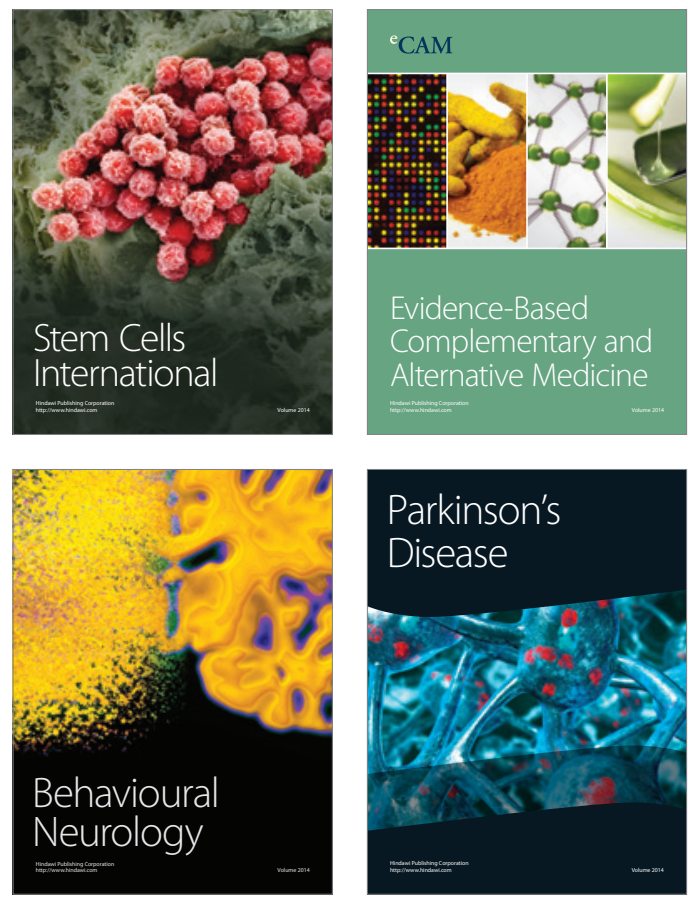
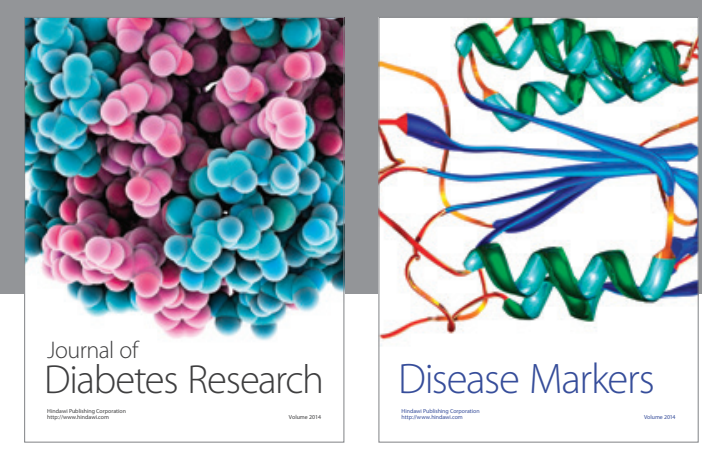

Disease Markers
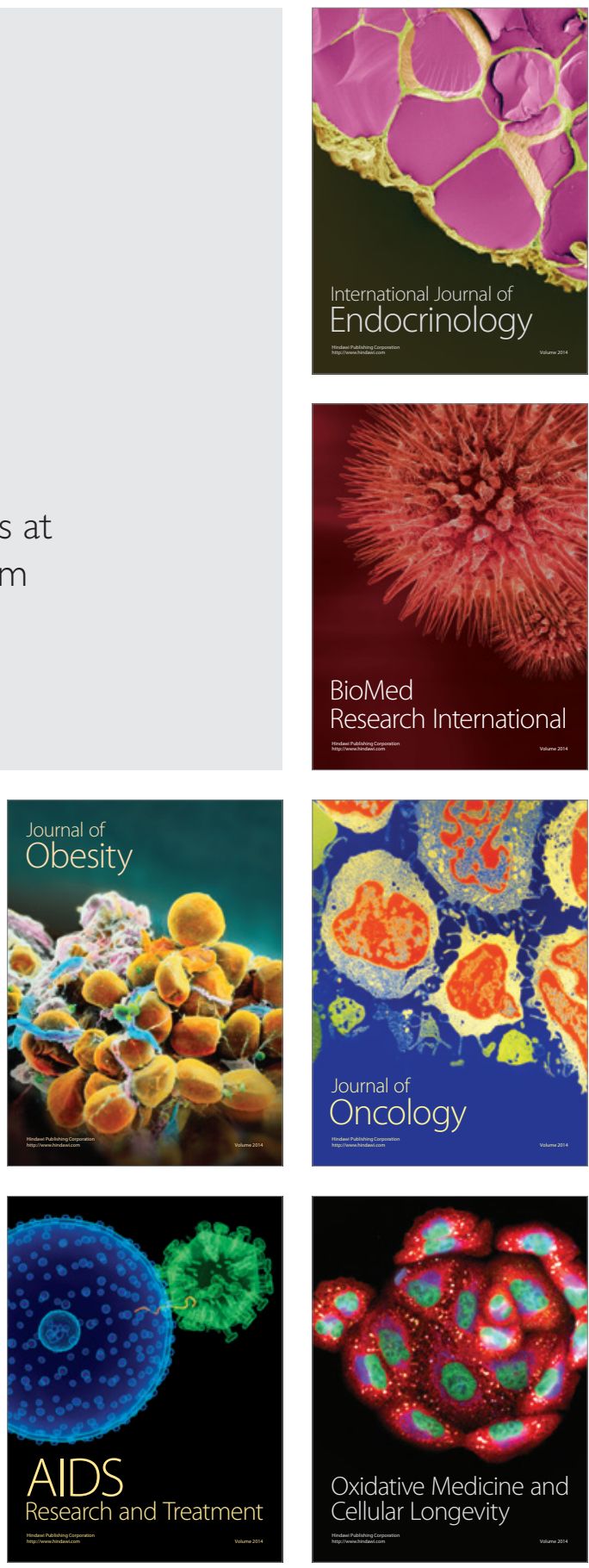